\title{
Numerical Evidence for a Conjecture in Real Algebraic Geometry
}

\author{
Jan Verschelde
}

\section{CONTENTS}

1. Introduction

2. Testing a Conjecture of Boris and Michael Shapiro

3. Why We Need New Homotopies

4. SAGBI Homotopies

5. The Pole Placement Problem

6. Symbolic-Numeric Calculations

7. Available Software and Execution Times

8. Conclusions

Acknowledgements

References

Supported by a post-doctoral fellowship at the Mathematical Sciences Research Institute, Berkeley, USA. Supported in part by NSF under grant DMS-9804846 at Michigan State University.

AMS Subject Classification: 14M15, 65H10,68Q40, 93B55.

Keywords: polynomial system, numerical Schubert calculus, SAGBI homotopy, polyhedral continuation, flat deformation, cheater's homotopy, pole placement problem, control theory.
Homotopies provide computational evidence for a challenging instance of a conjecture about whether all solutions are real. By a homotopy we mean a family of polynomial systems that describes algebraically the geometric transition from an easier configuration in special position into the general configuration for the problem we want to solve. The solutions to our problem lie at the end of the solution paths we trace with numerical continuation methods starting at the solutions of the easier, special problem. The numerical difficulties are overcome if we work in the true synthetic spirit of the Schubert calculus, selecting the numerically most favorable equations to represent the geometric problem. Since a well-conditioned polynomial system allows perturbations on the input data without destroying the reality of the solutions we obtain not just one instance, but a whole manifold of systems that satisfy the conjecture. Also an instance that involves totally positive matrices has been verified. The optimality of the solving procedure is a promising first step towards the development of numerically stable algorithms for the pole placement problem in linear systems theory.

\section{INTRODUCTION}

Solving a polynomial system numerically means computing approximations to all isolated solutions of the system. Having an approximate root, as in [Blum et al. 1998], implies that Newton's method doubles its accuracy in each step. Homotopy continuation methods provide paths to all isolated approximate roots. The references [Morgan 1987; Li 1997; Cox et al. 1998] treat polynomial homotopies respectively from within the fields of engineering, numerical analysis and computational algebraic geometry. Path-following methods are described in [Allgower and Georg 1990; 1997].

Optimal homotopies for solving polynomial systems arising in the Schubert calculus of enumerative geometry were proposed by Birk Huber, Frank Sottile and Bernd Sturmfels in [Huber et al. 1998]. 
These homotopies are optimal in that every path leads to a solution when applied to a generic problem instance, whereas the standard homotopies force one to trace many diverging solution paths.

The results described in this article were obtained with SAGBI homotopies [Huber et al. 1998]. Their implementation leads to a three-stage solver concatenating polyhedral continuation, flat deformation and cheater's homotopy. Polyhedral homotopies [Huber and Sturmfels 1995; Verschelde et al. 1994] are optimal for systems with generic coefficients. The flat deformations defined by the SAGBI homotopies transform a generic intersection problem into a polynomial system with generic coefficients. Cheater's homotopy [ $\mathrm{Li}$ et al. 1989; $\mathrm{Li}$ and Wang 1992], or coefficient-parameter polynomial continuation [Morgan and Sommese 1989], ensures that singularities may only occur at the end of the solution paths. This last stage is invoked when solving a specific real problem instance.

The specific problem we wish to solve represents the geometric problem of enumerating all $p$-planes that meet $m p$ given $m$-planes in $\mathbb{C}^{m+p}$. When those given $m$-planes are in general position, the number of solution $p$-planes is finite. An explicit formula to count the solutions was derived by Schubert [1891]. Brocket and Byrnes [1981] showed that every solution to this classical problem of enumerative geometry corresponds to a feedback law to control an $m$-input, $p$-output machine whose evolution is described by a linear system. This establishes the geometric interpretation of the so-called pole placement problem in linear systems theory [Byrnes 1989]. Every solution path defined by the homotopies developed in [Huber et al. 1998] ends at a $p$-plane and corresponds to a feedback law. The optimality of the homotopies is a promising first step to providing numerical algorithms for the pole placement, formulated as one of the open problems in [Rosenthal and Willems 1999].

The purpose of this paper is to report on verified large instances of some conjectures of Boris and Michael Shapiro. They proposed specific choices for the input $m$-planes and conjectured that for those specific input data all solution $p$-planes would be real. Having real solutions is important because the machines are specified with real data, and real feedback laws are required for control. Note that, if all feedback laws are real, then to control the machine it suffices to compute just one solution to the geometric problem, which can simply be done by following one single solution path. To verify the conjectures, we have to find all solutions to the polynomial systems, but for engineering applications [Kailath 1980] finding just one solution is enough. We refer to [Faugère et al. 1998; Rosenthal and Sottile 1998; Sottile 2000b] for other tests and related work on these conjectures. Note that Sottile [1999] developed an asymptotic choice of inputs for which he proved that all solutions are real.

The type of polynomial system that needs to be solved is presented in the next section, followed by a survey on standard root-counting methods. Thereafter come implementational aspects for the homotopies and a derivation of the equations in the pole placement problem. A report on the main numerical difficulties and solutions is given in the sixth section. The last part of the paper contains a short description of the freely available software package PHC developed by the author. Execution times are listed, illustrating the performance of the methods.

\section{TESTING A CONJECTURE OF BORIS AND MICHAEL SHAPIRO}

Our problem instance is encoded as $(m, p)=(2,8)$; see [Faugère et al. 1998; Rosenthal and Sottile 1998; Sottile 2000b; 1999] for other cases and related work. The formulation used here is as a problem in enumerative geometry.

Given 16 2-planes osculating a rational normal curve, we look for 8-planes in $\mathbb{C}^{10}$ that meet these 16 given 2-planes nontrivially. The intersection is nontrivial if and only if, for $i=1,2, \ldots, 16$,

$$
\begin{gathered}
f_{i}(\boldsymbol{x})=\operatorname{det}\left[\begin{array}{cccccc}
1 & 0 & x_{11} & x_{12} & \cdots & x_{18} \\
s_{i} & 1 & x_{21} & x_{22} & \cdots & x_{28} \\
s_{i}^{2} & 2 s_{i} & 1 & 0 & \cdots & 0 \\
s_{i}^{3} & 3 s_{i}^{2} & 0 & 1 & \cdots & 0 \\
\vdots & \vdots & \vdots & \vdots & \ddots & \vdots \\
s_{i}^{9} & 9 s_{i}^{8} & 0 & 0 & \cdots & 1
\end{array}\right] \\
=0 .
\end{gathered}
$$

The given 2-planes are spanned by the first two columns of the matrix; the second column is the derivative of the first. This makes it tangent to a space curve, whence we call it 'osculating'. 
The last eight columns of (2-1) span the unknown 8 -planes. Local coordinates are chosen fixing the lower-right block of the matrix as the identity matrix. The 16 unknowns $x_{i j}$ are determined by 16 equations that come from choosing 16 distinct $s_{i^{-}}$ values and expanding the determinant $(2-1)$. This distinct choice implies to have a generic problem instance with a finite number of solutions.

Schubert [1891] computed that there are generically 1430 solutions to this problem. The ShapiroShapiro conjecture is that all solutions to the polynomial system $(2-1)$ are real. Another version of this conjecture involving totally positive matrices is described at the end of section six.

\section{WHY WE NEED NEW HOMOTOPIES}

The standard homotopies perform poorly when applied directly to our problem because the bounds on the number of solutions are too high. We now review various standard root-counting methods, which give a priori bounds that determine the number of paths in the homotopies.

Definition 3.1. The total degree $D$ of $F(\boldsymbol{x})=\mathbf{0}$ is

$$
D=\prod_{i=1}^{n} \operatorname{deg}\left(f_{i}\right)
$$

Theorem 3.2 (Bézout). $F(\boldsymbol{x})=\mathbf{0}$ has at most $D$ isolated complex solutions, counted with multiplicities.

In [Morgan 1987], the theorem is proved by differential geometry. A proof based on projective elimination theory appears in [Cox et al. 1997]. In [Blum et al. 1998] we find another proof with a treatment of the complexity.

Expanding (2-1) we obtain 16 quadratic polynomials. Applying Bézout's theorem gives $D=2^{16}=$ 65,536 . A homotopy based on this bound requires thus the tracing of 65,536 solution paths with only 1430 (= Schubert's bound) converging ones.

The application of multihomogeneous homotopies was introduced in [Morgan and Sommese 1987] and has been applied with great success to various problems in mechanism design; see [Wampler et al. 1990; 1992], for example.

Definition 3.3. Denote $\left\{x_{1}, x_{2}, \ldots, x_{n}\right\}$ by $X$. Consider $S \subseteq X$. The degree $\operatorname{deg}(f, S)$ of $f$ in $S$ is $\operatorname{deg}\left(f\left(\left.\boldsymbol{x}\right|_{x_{i}=1, i \notin S}\right)\right)$.
Definition 3.4. Let $Z=\left\{Z_{1}, Z_{2}, \ldots, Z_{k}\right\}$ be a partition of $X$. For $F$, the degree matrix $M \in \mathbb{N}^{n \times k}$ is defined as $M_{i j}:=\operatorname{deg}\left(f_{i}, Z_{j}\right)$. The $k$-homogeneous Bézout number $B$ is the permanent of $M$.

The permanent of the degree matrix $M$ counts the number of solutions of a random linear-product start system. Every equation is the product of $\# Z$ factors. The $j$-th factor for the $i$-th equation is the product of $M_{i j}$ linear equations in the unknowns of $Z_{j}$, with random coefficients.

Theorem 3.5. For any partition $Z$ of $X$ we have $k=\# Z$. The corresponding $k$-homogeneous Bézout number $B$ bounds the number of isolated complex solutions of $F(\boldsymbol{x})=\mathbf{0}$, counted with multiplicities.

A multihomogeneous homotopy (see [Morgan and Sommese 1987]) reaches all isolated solutions of a polynomial system.

Different partitions give different Bézout numbers $B$. A partition with the minimal $B$ is hard to find in general. See [Wampler 1992] for exhaustive search algorithms. In applications the optimal partition follows from the meaning of the variables. Here we naturally group according to rows or columns. For the $(2,8)$-case, the partition $\left\{\left\{x_{11}, x_{12}, \ldots, x_{18}\right\}\right.$, $\left.\left\{x_{21}, x_{22}, \ldots, x_{28}\right\}\right\}$ gives $B=12,870$.

Polyhedral methods have led to a computational breakthrough in solving polynomial systems. See [Sturmfels 1998] for developments and open problems on counting roots with polytopes.

Definition 3.6. Consider $f(\boldsymbol{x})=\sum_{\boldsymbol{a} \in A} c_{\boldsymbol{a}} \boldsymbol{x}^{\boldsymbol{a}}$, with $c_{\boldsymbol{a}} \in \mathbb{C}$ and $\boldsymbol{x}^{\boldsymbol{a}}=x_{1}^{a_{1}} x_{2}^{a_{2}} \cdots x_{n}^{a_{n}}$. The set $A=\{\boldsymbol{a} \in$ $\left.\mathbb{Z}^{n} \mid c_{\boldsymbol{a}} \neq 0\right\}$ is the support of $f$. The convex hull of $A$ is the Newton polytope of $f$.

Definition 3.7. Let $\mathcal{P}=\left(P_{1}, P_{2}, \ldots, P_{n}\right)$ be a tuple of polytopes. The mixed volume $V_{n}(\mathcal{P})$ of $\mathcal{P}$ is

$$
V_{n}(\mathcal{P})=\sum_{I \subseteq\{1,2, \ldots, n\}}(-1)^{n-\# I} \operatorname{vol}_{n}\left(\sum_{i \in I} P_{i}\right),
$$

where $\operatorname{vol}_{n}$ is the usual volume.

Theorem 3.8 (Bernshteĭn). A system $F(\boldsymbol{x})=\mathbf{0}$ with Newton polytopes $\mathcal{P}$ has no more than $V_{n}(\mathcal{P})$ isolated complex solutions in $\left(\mathbb{C}^{*}\right)^{n}$ counted with multiplicities (where $\left.\mathbb{C}^{*}=\mathbb{C} \backslash\{0\}\right)$. 


\begin{tabular}{|c|c|c|c|c|c|c|c|c|c|c|c|c|}
\hline \multirow[b]{2}{*}{$p$} & \multicolumn{8}{|c|}{$m=2$} & \multicolumn{4}{|c|}{$m=3$} \\
\hline & 2 & 3 & 4 & 5 & 6 & 7 & 8 & 9 & 2 & 3 & 4 & 5 \\
\hline$D$ & 16 & 64 & 256 & 1024 & 4096 & 16384 & 65536 & 262144 & 64 & 19683 & 531441 & 14348907 \\
\hline$B$ & 6 & 20 & 70 & 252 & 924 & 3432 & 12870 & 48620 & 20 & 1680 & 34650 & 756756 \\
\hline$V$ & 4 & 17 & 66 & 247 & 918 & 3425 & 12862 & 48611 & 17 & 642 & 22148 & $?$ \\
\hline$N$ & 2 & 5 & 14 & 42 & 132 & 429 & 1430 & 4862 & 5 & 42 & 462 & 6006 \\
\hline
\end{tabular}

TABLE 1. Standard root counts and the actual number of $\operatorname{roots} N$ for various $(m, p)$-systems.

The proof in [Bernshtel̆ 1975] is constructive, and was implemented in [Verschelde et al. 1994]. See also [Cox et al. 1998].

We see from $(2-1)$ that all supports are equal, so $P=P_{i}$ for $i=1,2, \ldots, n$, and then $V_{n}(\mathcal{P})=$ $n ! \operatorname{vol}_{n}(P)$, which is the case of Kushnirenko's theorem [1976]. The direct application of Bernshteinn's theorem leads to a homotopy with $\operatorname{vol}_{n}(P)=12,862$ solution paths.

For this $(2,8)$-instance, only 1430 solution paths converge using homotopies based on the above root counts, as predicted by Schubert's formula [1891]. Table 1 summarizes the performance of the root counts. More such tables can be found in [Sottile 2000b].

If $p \leq m$, separating the unknowns that belong to different rows gives the minimal $B$. Otherwise, we better group unknowns that belong to the same columns. For $m=2, B$ is remarkably close to $V$. For $m=3$, the gap widens. The calculation of $B$ requires a permanent computation which is a $\# P$ hard problem. This means that there is no nondeterministic polynomial time algorithm available to solve this problem for general dimensions. Despite this, the algorithms in [Wampler 1992] outperform the volume computation.

\section{SAGBI HOMOTOPIES}

SAGBI homotopies are one of the three homotopies proposed in [Huber et al. 1998]. Here we summarize the method emphasizing the algorithmic aspects. The general principles are illustrated with a running example. At each stage characteristics in solving the $(2,8)$-case are listed.

To implement SAGBI homotopies we concatenate three different homotopies, see Figure 1. Table 2 defines the polynomial systems at each stage. Complex arithmetic in this concatenation is necessary because of singularities that otherwise may occur. In solving several real problem instances, we recycle the solutions of one generic complex problem using cheater's homotopy.

Example 4.1 (Running example). The system for

$$
(m, p)=(2,2)
$$

consists of four equations:

$$
f_{i}(\boldsymbol{x})=\operatorname{det}\left[\begin{array}{cccc}
1 & 0 & x_{11} & x_{12} \\
s_{i} & 1 & x_{21} & x_{22} \\
s_{i}^{2} & 2 s_{i} & 1 & 0 \\
s_{i}^{3} & 3 s_{i}^{2} & 0 & 1
\end{array}\right]=0,
$$

for $i=1,2,3,4$. To create a specific real problem we choose four different values for $s_{i}$ and apply Laplace expansion in terms of 2 -by- 2 minors. The polynomials in the SAGBI homotopy are

$$
\begin{aligned}
& \hat{f}_{i}(\boldsymbol{x}, t)=C_{34}^{(i)}\left(x_{11} x_{22}-t x_{12} x_{21}\right)-C_{24}^{(i)}\left(-x_{12}\right) \\
& +C_{23}^{(i)} x_{11}+C_{14}^{(i)}\left(-x_{22}\right)-C_{13}^{(i)} x_{21}+C_{12}^{(i)}=0,
\end{aligned}
$$

for $i=1,2,3,4$. The lower indices in the coefficients $C_{k l}^{(i)}$ refer to the choice of rows of the matrix in the elaboration of (4-1). The SAGBI homotopy starts at $t=0$ and ends at $t=1$. At $t=1$ the generic complex problem has coefficients $C_{k l}^{(i)}$ which are 2by-2 minors of a 4 -by- 2 matrix of random complex coefficients.

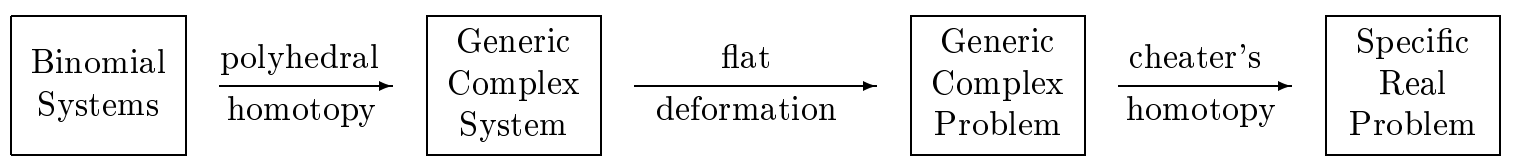

FIGURE 1. Concatenation of three homotopies. The central part in the chain is the SAGBI homotopy. 


\begin{tabular}{|lll|}
\hline Binomial System & $:$ & exactly two monomials in every equation \\
Generic Complex System & $:$ & coefficients are randomly chosen complex numbers \\
Generic Complex Problem & $:$ & problem with randomly chosen complex planes \\
Specific Real Problem & $:$ & real osculating planes as in the conjecture \\
\hline
\end{tabular}

TABLE 2. The end points in the homotopies are four different types of polynomial systems.

Our running example is a classical problem in enumerative geometry. Note that 2-planes in $\mathbb{C}^{4}$ correspond to lines in $\mathbb{P}^{3}$. The question is then to find all lines that intersect four given lines nontrivially. In general, two lines satisfy this condition; see Figure 2, where the positive real orthant of projective 3 -space corresponds to the interior of the tetrahedron. Note that this problem is fully real: both the input and output lines are real lines.

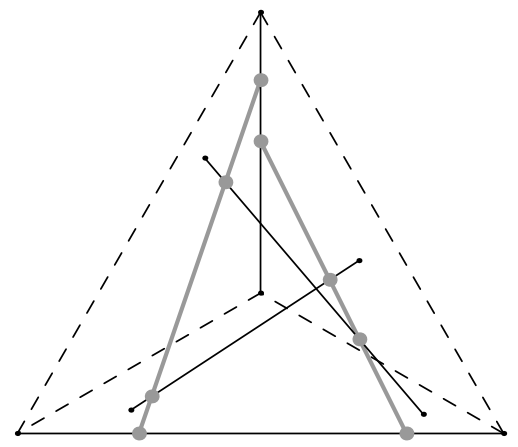

FIGURE 2. The case $m=2=p$. Given four lines (drawn thin and black) in general position there are exactly two lines that intersect all four (thicker and gray).

The special position of two of the four input lines is adapted from the Pieri homotopy algorithm $[\mathrm{Hu}-$ ber et al. 1998]. Another choice of local coordinates, unlike those in (4-1), for the output planes enables to solve this instance.

\section{A. Newton Polytopes and Polyhedral Continuation}

To compute the mixed volume we need a subdivision of the polytopes. A regular subdivision is obtained by lifting the polytopes into a higher-dimensional space and projecting the facets of the lower hull of the sum of the lifted polytopes down to the original space. The key idea of [Huber and Sturmfels 1995] is that this lifting induces the so-called polyhedral homotopy, starting at binomial systems whose Newton polytopes match the cells in the subdivision. We refer to [Li 1997] for numerical aspects.
For our problem we compute the volume of one Newton polytope, for which the dynamic lifting algorithm [Verschelde et al. 1996] is well-suited. This algorithm incrementally updates the triangulation each time selecting the lowest possible lifting value for the added point. The induced homotopies have a minimal power in the continuation parameter. For the $(2,8)$-case, the highest lifting value is 133 .

Example 4.2 (continuation of Example 4.1). The polyhedral homotopy that is induced by the dynamic lifting algorithm consists of the equations

$$
\begin{aligned}
\hat{g}_{i}(\boldsymbol{x}, t)=c_{1}^{(i)} x_{11} & x_{22}+c_{2}^{(i)} x_{11} t+c_{3}^{(i)} x_{12} \\
& +c_{4}^{(i)} x_{21}+c_{5}^{(i)} x_{22}+c_{6}^{(i)}=0,
\end{aligned}
$$

for $i=1,2,3,4$. The coefficients $c_{j}^{(i)}$ are random complex constants. The triangulation has two cells and correspondingly there are two homotopies. The first homotopy equals (4-3). At $t=1$ we have the system we want to solve. At $t=0$ we have a system of four equations, each with exactly five terms. By Gaussian elimination we obtain a binomial system that can be solved efficiently [Verschelde et al. 1994]. So at $t=0$ we know the solutions and we can start following the paths defined by the homotopy (4-3).

Ordering the variables as $\left(x_{11}, x_{12}, x_{21}, x_{22}, t\right)$, the support of (4-3) is the lifted point configuration $\hat{A}=$ $\{(1,0,0,1,0),(0,1,0,0,0),(1,0,0,0,1),(0,0,0,1,0)$, $(0,0,1,0,0),(0,0,0,0,0)\}$. The other cell in the triangulation consists of the points $\hat{\boldsymbol{a}} \in \hat{A}$ for which the inner product $\langle\hat{\boldsymbol{a}}, \boldsymbol{v}\rangle$ with $\boldsymbol{v}=(-1,0,0,1,1)$ is minimal. To set up the second homotopy we replace $\boldsymbol{x}^{\boldsymbol{a}}$ by $\boldsymbol{x}^{\boldsymbol{a}} t^{\langle\hat{\boldsymbol{a}}, \boldsymbol{v}\rangle}$ :

$$
\begin{aligned}
\hat{h}_{i}(\boldsymbol{x}, t)=c_{1}^{(i)} x_{11} x_{22}+ & c_{2}^{(i)} x_{11}+c_{3}^{(i)} x_{12} \\
& +c_{4}^{(i)} x_{21}+c_{5}^{(i)} x_{22} t+c_{6}^{(i)}=0,
\end{aligned}
$$

for $i=1,2,3,4$. At $t=1$ we have the system we want to solve. At $t=0$ we can again reduce the system to a binomial system whose solutions are the starting points of the paths defined by the homotopy in the preceding equation. 
We have found all roots for the system we wanted to solve since $(0,0,0,0,1)$ and $(-1,0,0,1,1)$ are the only two vectors (unique upon scaling) for which the selection of points with minimal inner product leads to a cell with nonzero volume and a homotopy that has solutions in $\left(\mathbb{C}^{*}\right)^{4}$ at $t=0$.

The triangulation for the $(2,8)$-case consists of 1430 simplices because the Newton polytope is the order polytope [Sturmfels 1996] which is unimodular.

\section{B. Flat Deformations arising from Gröbner and SAGBI Bases}

The first homotopy of the three presented in [Huber et al. 1998] is the Gröbner homotopy. Although its efficiency is inferior compared to the SAGBI homotopy, it helps understanding the latter.

Gröbner basics [Sturmfels 1996] teach us that for any term order $\prec$ and ideal $I$, there exists a weight vector $\boldsymbol{w} \in \mathbb{N}^{n}$ defining a monomial order $\prec_{\boldsymbol{w}}$ (as: $\boldsymbol{x}^{\boldsymbol{a}} \prec_{\boldsymbol{w}} \boldsymbol{x}^{\boldsymbol{b}} \Longleftrightarrow\langle\boldsymbol{a}, \boldsymbol{w}\rangle>\langle\boldsymbol{b}, \boldsymbol{w}\rangle$ or $\langle\boldsymbol{a}, \boldsymbol{w}\rangle=\langle\boldsymbol{b}, \boldsymbol{w}\rangle$ and $\left.\boldsymbol{x}^{\boldsymbol{a}} \prec_{\text {lex }} \boldsymbol{x}^{\boldsymbol{b}}\right)$ that induces the same initial ideal: in $_{\prec}(I)=$ in $_{\prec_{w}}(I)$. In [Eisenbud 1995], given a Gröbner basis, a homotopy is defined to deform the zero set of the ideal $I$ into the zero set of in $_{\prec w}(I)$. In particular, the parameter $t$ in the flat deformation is introduced substituting each $x_{i}$ by $x_{i} t^{\boldsymbol{w}\left(x_{i}\right)}$, where $\boldsymbol{w}\left(x_{i}\right)$ is the weight of $x_{i}$. Hereby $\boldsymbol{x}^{\boldsymbol{a}}$ is replaced by $\boldsymbol{x}^{a} t^{\langle\boldsymbol{a}, \boldsymbol{w}\rangle}$. All systems in that homotopy have the same initial ideal, whence the same Hilbert function, whence the same structure of the zero set for all $t$.

The unknowns in the Gröbner homotopy are $p$-by$p$ minors, denoted by brackets. A bracket is a vector of indices to the rows that have been selected in constructing the minor. The monomials are products of brackets. We see monomials as tableaux that have brackets in their rows. A tableau is standard if its columns are sorted, otherwise it is nonstandard. The straightening algorithm provides relations to rewrite a nonstandard tableau as a linear combination of standard ones. The combinatorics of tableaux and the relation with the Schubert calculus are treated in [Fulton 1997].

Example 4.3 (a straightening syzygy). A 2-by-2 minor that selects the $i$-th and $j$-th row of a matrix, $i<j$, is represented by the bracket $[i j]$. Expanding the determinant in (4-4) along the first row, we see that we always obtain two equal columns. With Laplace expansion into 2-by-2 minors we obtain in bracket notation a straightening syzygy. Thus,

$$
\operatorname{det}\left[\begin{array}{cccc}
x_{11} & x_{12} & 0 & 0 \\
x_{21} & x_{22} & x_{21} & x_{22} \\
x_{31} & x_{32} & x_{31} & x_{32} \\
x_{41} & x_{42} & x_{41} & x_{42}
\end{array}\right] \equiv 0
$$

is equivalent to

$$
\left[\begin{array}{ll}
1 & 2
\end{array}\right]\left[\begin{array}{ll}
3 & 4
\end{array}\right]-\left[\begin{array}{ll}
1 & 3
\end{array}\right]\left[\begin{array}{ll}
2 & 4
\end{array}\right]+\left[\begin{array}{ll}
1 & 4
\end{array}\right]\left[\begin{array}{ll}
2 & 3
\end{array}\right]=0 .
$$

We can rewrite the nonstandard monomial [1 4 1 $]\left[\begin{array}{ll}2 & 3\end{array}\right]$ as a linear combination of standard ones.

The defining ideal of the Grassmann manifold is generated by all straightening syzygies needed to rewrite all possible nonstandard tableaux. Sturmfels [1993] proved that these rewriting rules constitute a Gröbner basis for the term order that selects the monomials corresponding to the nonstandard tableaux as the leading ones. In [Huber et al. 1998] we find a recipe to assign weights and to set up the flat deformation. The initial ideal is squarefree so that the start solutions are regular. Except for coefficients that belong to an algebraic set, all solutions are regular.

Example 4.4 (continuation of Example 4.2). The Gröbner homotopy consists of five equations, homogeneous in the six brackets, namely

$$
\left[\begin{array}{ll}
1 & 4
\end{array}\right]\left[\begin{array}{ll}
2 & 3
\end{array}\right]-\left[\begin{array}{ll}
1 & 3
\end{array}\right]\left[\begin{array}{ll}
2 & 4
\end{array}\right] t+\left[\begin{array}{ll}
1 & 2
\end{array}\right]\left[\begin{array}{ll}
3 & 4
\end{array}\right] t^{2}=0
$$

and

$$
\begin{aligned}
C_{34}^{(i)}\left[\begin{array}{ll}
1 & 2
\end{array}\right] & -C_{24}^{(i)}\left[\begin{array}{ll}
1 & 3
\end{array}\right]+C_{23}^{(i)}\left[\begin{array}{ll}
1 & 4
\end{array}\right] \\
& +C_{14}^{(i)}\left[\begin{array}{ll}
2 & 3
\end{array}\right]-C_{13}^{(i)}\left[\begin{array}{ll}
2 & 4
\end{array}\right]+C_{12}^{(i)}\left[\begin{array}{ll}
3 & 4
\end{array}\right]=0,
\end{aligned}
$$

for $i=1,2,3,4$. The first of these equations comes from (4-4). The coefficients $C_{k l}^{(i)}$ are minors selecting the $k$-th and $l$-th row of a 4 -by- 2 matrix with random complex coefficients. At $t=0$, there are two start solutions.

Note that the parameter $t$ only appears in the equation that defines the Grassmann manifold. The linear equations of the intersection condition remain invariant under the flat deformation.

In the $(2,8)$-case, the coefficients are 2 -by- 2 minors and the unknowns are brackets representing 8-by- 8 minors, $\left(\begin{array}{c}10 \\ 8\end{array}\right)=45$ unknowns in total. Expanding (2-1) leads to a 16 -by-16 linear system in the brackets constrained by 210 quadratic equations 
that define Grassmann manifold. The Gröbner homotopy solves the $(2,8)$-problem as an overdetermined system of 226 equations in 45 unknowns.

When the minors are expanded in terms of the $x_{i j}$ 's the equations that define the Grassmann manifold are automatically satisfied. Therefore we are left with fewer equations than in the Gröbner homotopy, which explains the superior efficiency of the SAGBI homotopy. In particular, for the $(2,8)$-case we are left with 16 equations in 16 unknowns.

After the expansion in the $x_{i j}$ 's we no longer have an ideal but polynomials that form a subalgebra in the polynomial ring $\mathbb{C}\left[x_{i j}\right]$. SAGBI stands for Subalgebra Analogue to Gröbner Bases for Ideals. The term order we use selects as leading monomial the product of elements on the diagonal of the matrices. In [Sturmfels 1996] flat deformations are extended to SAGBI bases. The general recipe for the flat deformation in [Huber et al. 1998] is given by substituting $x_{i j}$ by $x_{i j} t^{(i-1)(p-j)}$ and dividing out the lowest power of $t$, minor per minor. The leading monomials with this term order have minimal powers of $t$ and are products of diagonal elements in the minors.

Example 4.5 (continuation of Example 4.4). The SAGBI homotopy (4-2) in determinantal form is

$$
f_{i}(\boldsymbol{x})=\operatorname{det}\left[\begin{array}{cccc}
c_{11}^{(i)} & c_{12}^{(i)} & x_{11} & x_{12} \\
c_{21}^{(i)} & c_{22}^{(i)} & x_{21} t & x_{22} \\
c_{31}^{(i)} & c_{32}^{(i)} & 1 & 0 \\
c_{41}^{(i)} & c_{42}^{(i)} & 0 & 1
\end{array}\right]
$$

for $1,2,3,4$, where the coefficients $c_{k l}^{(i)}$ are random complex constants. The brackets are expanded as follows:

$$
\begin{aligned}
& {\left[\begin{array}{ll}
1 & 2
\end{array}\right] \leftarrow x_{11} x_{22}-x_{21} x_{12} t \quad\left[\begin{array}{ll}
2 & 3
\end{array}\right] \leftarrow-x_{22}} \\
& {\left[\begin{array}{ll}
1 & 3
\end{array}\right] \leftarrow-x_{12} \quad\left[\begin{array}{ll}
2 & 4
\end{array}\right] \leftarrow x_{21}} \\
& {\left[\begin{array}{ll}
1 & 4
\end{array}\right] \leftarrow x_{11} \quad[3 \quad 4] \leftarrow 1}
\end{aligned}
$$

Substituting the brackets into the linear equations of (4-5) and (4-6) gives the SAGBI homotopy (4-2).

The SAGBI homotopies are nonlinear in $t$. For the $(2,8)$-case, the highest power of $t$ equals 7 .

\section{C. The Cheater's Homotopy}

In [ $\mathrm{Li}$ et al. 1989] the so-called cheater's homotopy was presented to solve repeatedly a polynomial sys- tem with coefficients as functions of parameters for several instances of these parameters. The procedure assumes that one has solved the polynomial system once (this is the cheating part) for a generic complex choice of the parameters. See [Morgan and Sommese 1989] for a similar idea.

The parameters in our problem are the minors from the $m$-planes. To avoid repeated evaluation of minors we apply the result of [Li and Wang 1992]. The start system is a problem instance for a generic choice of the parameters $\boldsymbol{c}^{0} \in \mathbb{C}^{k}$. For any $\boldsymbol{c} \in \mathbb{C}^{k}$, the following homotopy is guaranteed to reach all solutions of $F(\boldsymbol{c}, \boldsymbol{x})=\mathbf{0}$ :

$$
\begin{aligned}
H(\boldsymbol{c}, t) & =F\left((1-[t-t(1-t) \gamma]) \boldsymbol{c}^{0}+(t-t(1-t) \gamma) \boldsymbol{c}, \boldsymbol{x}\right) \\
& =\mathbf{0},
\end{aligned}
$$

for $t \in[0,1]$ and $\gamma \in \mathbb{C}$. This homotopy avoids the evaluation of the parameters during path following.

Example 4.6 (continuation of Example 4.5). Take the homotopy $H(\boldsymbol{x}, t)=\mathbf{0}$ in (4-8). At $t=0$, the parameters $\boldsymbol{c}^{0}$ consist of all 2-by-2 minors $C_{k l}^{(i)}$ of a 4-by-2 random complex matrix. This system has been solved by the SAGBI homotopy. At $t=1$, the parameters $c$ consist of the maximal minors of the first two columns of the matrix in $(4-1)$. The two solution paths converge to two distinct real solutions that span 2-planes intersecting the two given 2-planes nontrivially.

Using cheater's homotopy, the conjecture can be tested systematically, deforming so that every system in the family is a real problem instance. If singular solutions occur where two real solution paths join into a complex conjugate pair of solutions, then the conjecture is false.

\section{THE POLE PLACEMENT PROBLEM}

In this section we describe how to translate the pole placement problem of linear systems theory [Byrnes 1989; Rosenthal and Schumacher 1997] into the geometric formulation (2-1). This connection was established in [Brockett and Byrnes 1981]. First we give a classical example [Kailath 1980] of control.

In Figure 3 we consider the balancing of a pointer. The controller has to bring the pointer back to upright position after a slight deviation. In deriving the evolution equation we assume that all mass $\boldsymbol{m}$ 
is located at the top of the pointer. Furthermore, the controller only moves in the direction of the $x$ axis. To obtain a linear system, we assume that the deviation angle $\alpha$ is small.

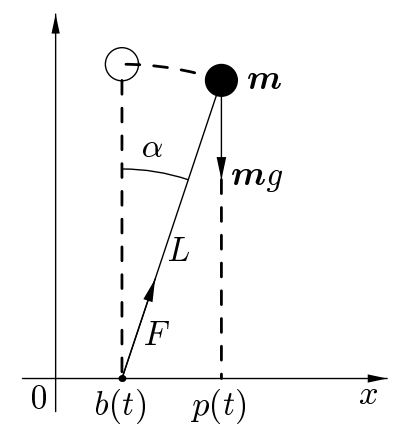

FIGURE 3. Balancing a pointer of length $L$ with mass $\boldsymbol{m}$ at top.

If the pointer has base point $b(t)$, the projection of the top on the $x$-axis has position $p(t)$ :

$$
p(t)=b(t)+L \sin (\alpha(t)) \approx b(t)+L \alpha(t) .
$$

The projection of the gravitational force onto the pointer is $\boldsymbol{m} g \cos (\alpha(t)) \approx \boldsymbol{m} g$, as $\cos (\alpha(t)) \approx 1$ for $\alpha(t) \approx 0$. Denote by $F$ the magnitude of the force acting at the bottom end of the pointer. To compensate for gravity, $F=\boldsymbol{m} g$. The $x$-component of $F$ is $F_{x}(t)=\boldsymbol{m} \ddot{p}(t)$, so:

$$
\boldsymbol{m} \ddot{p}(t)=\boldsymbol{m} g \sin (\alpha(t)) \approx \boldsymbol{m} g \alpha(t) .
$$

Elimination of the position of the projected center of mass yields $g \alpha(t)=\ddot{b}(t)+L \ddot{\alpha}(t)$, or, equivalently,

$$
\ddot{\alpha}(t)=\frac{g}{L} \alpha(t)-\frac{\ddot{b}(t)}{L} .
$$

This second-order differential equation is equivalent to a system of first-order differential equations, introducing the states $\left(x_{1}, x_{2}\right)=(\alpha, \dot{\alpha})$. As input $u$, we take the impulse $u=\ddot{b}(t) / L$ and copy the states to the output $\left(y_{1}, y_{2}\right)$. Then the state-space description is given by the evolution equation

$$
\left[\begin{array}{c}
\dot{x}_{1} \\
\dot{x}_{2}
\end{array}\right]=\left[\begin{array}{cc}
0 & 1 \\
g / L & 0
\end{array}\right]\left[\begin{array}{l}
x_{1} \\
x_{2}
\end{array}\right]+\left[\begin{array}{r}
0 \\
-1
\end{array}\right][u],
$$

the output of the system

$$
\left[\begin{array}{l}
y_{1} \\
y_{2}
\end{array}\right]=\left[\begin{array}{ll}
1 & 0 \\
0 & 1
\end{array}\right]\left[\begin{array}{l}
x_{1} \\
x_{2}
\end{array}\right]
$$

and the input-to-output feedback

$$
[u]=\left[\begin{array}{ll}
-f_{1} & -f_{2}
\end{array}\right]\left[\begin{array}{l}
y_{1} \\
y_{2}
\end{array}\right] .
$$

In closed form, the system becomes

$$
\begin{aligned}
& {\left[\begin{array}{l}
\dot{x}_{1} \\
\dot{x}_{2}
\end{array}\right]=} \\
& \quad\left(\left[\begin{array}{cc}
0 & 1 \\
g / L & 0
\end{array}\right]+\left[\begin{array}{r}
0 \\
-1
\end{array}\right]\left[\begin{array}{ll}
-f_{1} & -f_{2}
\end{array}\right]\left[\begin{array}{ll}
1 & 0 \\
0 & 1
\end{array}\right]\right)\left[\begin{array}{l}
x_{1} \\
x_{2}
\end{array}\right] .
\end{aligned}
$$

To stabilize the system, all eigenvalues of the matrix represented by the expression in parentheses must all have a negative real part. If all eigenvalues have a pure imaginary part, the pointer oscillates. For eigenvalues with a positive real part, the system spins out of control. In this simple situation, we can determine the feedback laws by identification of the coefficients of the characteristic polynomial.

In general we consider a system with input $\boldsymbol{u} \in$ $\mathbb{R}^{m}$, output $\boldsymbol{y} \in \mathbb{R}^{p}$, and internal states $\boldsymbol{x} \in \mathbb{R}^{n}$, where $n=m+p$, whose evolution in time $t$ is governed by the first-order linear differential equation

$\dot{\boldsymbol{x}}(t)=A \boldsymbol{x}(t)+B \boldsymbol{u}(t) \quad$ with $A \in \mathbb{R}^{n \times n}, B \in \mathbb{R}^{n \times m}$, $\boldsymbol{y}(t)=C \boldsymbol{x}(t) \quad$ with $C \in \mathbb{R}^{p \times n}$,

$\boldsymbol{u}(t)=F \boldsymbol{y}(t) \quad$ with $F \in \mathbb{R}^{m \times p}$,

where the last part expresses the control of the input by constant output feedback $F$.

Substitution into the first of these three equations yields $\dot{\boldsymbol{x}}(t)=(A+B F C) \boldsymbol{x}(t)$, whose characteristic polynomial is

$$
\varphi(s)=\operatorname{det}\left(s I_{n}-A-B F C\right) .
$$

The roots $s_{i}(i=1,2, \ldots, n)$ of $\varphi(s)=0$ are the natural frequencies of the controlled system.

The pole placement problem is an inverse problem: given $A, B, C$, and $\varphi$ (determined by the frequencies $s_{i}$ ), compute the feedback laws $F$ that satisfy $\varphi\left(s_{i}\right)=0$, for $i=1,2, \ldots, n$.

We rewrite the characteristic equation $\varphi(s)=0$ in several stages. First, a determinantal identity shows that $\operatorname{det}\left(s I_{n}-A-B F C\right)=0$ is equivalent to

$$
\operatorname{det}\left[\begin{array}{ccc}
s I_{n}-A & 0 & -B \\
-C & I_{p} & 0 \\
0 & -F & I_{m}
\end{array}\right]=0,
$$

where $I_{p}$ and $I_{m}$ represent identity matrices of rank $p$ and $m$. Secondly, we see that this is further equivalent to

$$
\left.\operatorname{det}\left[\begin{array}{lll}
0 & -F & I_{m}
\end{array}\right)\left(\begin{array}{l}
X(s) \\
Y(s) \\
U(s)
\end{array}\right)\right]=0
$$


by applying the fact that

$$
\operatorname{det}\left[\begin{array}{c}
P(s) \\
M(s)
\end{array}\right]=c \operatorname{det}[M(s) Q(s)]
$$

for nonzero $c \in \mathbb{R}$, where

$$
P(s)=\left[\begin{array}{ccc}
s I_{n}-A & 0 & -B \\
-C & I_{p} & 0
\end{array}\right], \quad Q(s)=\left[\begin{array}{c}
X(s) \\
Y(s) \\
U(s)
\end{array}\right],
$$

and $P(s) Q(s)=0$. The $(n+p+m)$-by- $m$ polynomial matrix $Q(s)$ describes the the behavior of the system explicitly; that is, it gives for an $m$-input the new states, output and feedback. Finally, a simple elaboration transforms the characteristic equation of the pole placement problem into,

$$
\operatorname{det}\left[\begin{array}{cc}
U(s) & F \\
Y(s) & I_{m}
\end{array}\right]=0
$$

the familiar geometric form that we have used in solving the problem.

\section{SYMBOLIC-NUMERIC CALCULATIONS}

Singularities do not occur for generic complex problems. To solve the real problem, we choose orthogonal representations for the planes and obtain wellconditioned polynomial systems.

\section{A. Localizing before Expanding, Expanding before Cheating}

The equations (2-1) are already written in local coordinates. But any 8 -by- 8 submatrix using the last 8 columns can serve as unit matrix. Setting up the equations we better first fix this choice before expanding the determinants. Otherwise, see what happens in the $(2,8)$-case: every 8 -by- 8 minor has in its expansion $8 !=40,320$ terms and there are 45 such minors.

One can implement the cheater's homotopy in two ways. Either one can introduce the continuation parameter $t$ inside the matrix $(2-1)$ or one can do this after the expansion of the minors. The first way [Li et al. 1989] leads to homotopies that require the elaboration of determinants each time evaluation is needed. The second way [ $\mathrm{Li}$ and Wang 1992] uses (4-8) which does not require the evaluation of the parameters which leads to a much more efficient cheating procedure.
To illustrate this last point, the determinantal cheater's homotopy for the case $(4,2)$ requires 384 seconds of CPU time, versus 30 seconds for the homotopy which avoids the evaluation of determinants in every step.

\section{B. Using Chebyshev Polynomials as Basis}

Generating values for $s$ in equation (2-1) quickly leads to huge numbers when $s>1$ or very tiny numbers when $s<1$. The calculation of minors in the expansion of (2-1) amplifies this effect. We cannot take all values for $s$ too close to 1 because then the input planes lie too close to each other.

To approximate a function by a polynomial with minimal error the basis of Chebyshev polynomials (see [Gerald 1978], for example) is commonly used instead of the basis of standard monomials

$$
\left\{1, x, x^{2}, \ldots, x^{n}\right\} .
$$

The $n$-th Chebyshev polynomial $T_{n}(x)$ is defined as $\cos (n \arccos (x))$, whence $T_{n}(x) \in[-1,+1]$, for $x \in$ $[-1,+1]$. Therefore we obtain nice numbers for the coefficients in the polynomials generated by $(2-1)$. We show the equivalence of problem formulations by example.

Example 6.1 (reformulation of Example 4.1). Multiplying the 4-by-2 matrix by a nonsingular matrix gives an equivalent representation for the 2-plane. As the result of the multiplication

$$
\left[\begin{array}{rrrr}
1 & 0 & 0 & 0 \\
0 & 1 & 0 & 0 \\
-1 & 0 & 2 & 0 \\
0 & -3 & 0 & 4
\end{array}\right]\left[\begin{array}{cc}
1 & 0 \\
s & 1 \\
s^{2} & 2 s \\
s^{3} & 3 s^{2}
\end{array}\right]=\left[\begin{array}{cc}
1 & 0 \\
s & 1 \\
2 s^{2}-1 & 4 s \\
4 s^{3}-3 s & 12 s^{2}-3
\end{array}\right]
$$

we recognize the first four Chebyshev polynomials and their derivatives.

Note that this modification of the system $(2-1)$ does not change the problem, but only its representation as polynomial system. Actually, this reformulation gives rise to a wider class of polynomial systems whose solutions are all expected to be real.

Polynomials in the Chebyshev basis have more real roots on average than polynomials in the standard basis. In [Bharucha-Reid and Sambandham 1986] we read that a polynomial of degree $d$ with independent, normally distributed random coefficients written in the Chebyshev basis is expected to have 
$d / \sqrt{3}$ real roots, as $d \rightarrow \infty$, whereas this asymptotic bound is only $(2 / \pi) \log d$ for a random polynomial denoted in the standard basis. See [Cucker and Roy 1990] for more on the implication of the number of average real roots on complexity issues in computer algebra. In our case, Chebyshev polynomials lead to a numerically better formulation of a problem that is expected to have all its solutions real.

\section{C. Orthonormal Representations of the Planes}

Using Chebyshev polynomials suffices in case $m=2$, because the function values are bounded. However, it is hard to get bounded derivatives. In the $(8,2)$ case we must differentiate 7 times and compute 8by- 8 minors with the results of this differentiation. Without reformulation, only the cases where $m=2$ are numerically tractable.

The column vectors in orthogonal matrices form an orthogonal basis. Orthonormal matrices are the best conditioned representations of the input planes. The QR factorization [Golub and Van Loan 1996] of the matrix $A \in \mathbb{C}^{m \times n}$ rewrites $A$ as the product

$$
A=Q R
$$

where $Q \in \mathbb{C}^{m \times m}$ is orthonormal and $R \in \mathbb{C}^{m \times n}$ upper triangular. If $A$ has full column rank, then the first $n$ columns of $Q$ form an orthonormal basis for the space spanned by the columns of $A$. This factorization is computed by the QR algorithm [Golub and Van Loan 1996]. Adopting this representation, there is no difference between the cases $(3,4)$ and $(4,3)$.

Carrying this much further would be to develop a Newton's method for the Grassmann manifold, which would - instead of fixing the localization in advance - exploit the freedom of additional variables to work with orthogonal bases for the $p$-planes throughout the path following. As mentioned in [Edelman et al. 1999], there has never before been an explicit study of Newton's method on the Grassmann manifold. In our case we would have to approximate the Jacobian matrix to avoid dealing with the huge expanded polynomials before the localization. This is an interesting application of the secant method for which an $\alpha$-theory has recently been developed [Yakoubsohn 1999].

\section{D. Total Positivity}

Another conjecture of Boris and Michael Shapiro involves totally positive matrices, and is also described and tested in [Sottile 2000b]. A real matrix is totally positive [Ando 1987] if all its minors are positive. Upper triangular matrices are called totally positive when all minors are positive, except for those minors which vanish on all upper triangular matrices. For all upper triangular totally positive matrices $T$ considered here we assume $T_{i i}=1$.

The sequence of $m$-planes proposed in this ShapiroShapiro conjecture for which all solutions are expected to be real is defined as follows. The first $m$-plane can be spanned by any $(m+p)$-by- $m$ matrix. The next $m$-plane is generated by the last $m$ columns of the product of the previous $m$-plane with any $(m+p)$-by- $(m+p)$ random totally positive matrix. Repeat this generation until $m p m$-planes are obtained.

Since totally positive matrices have all their entries positive, a multiplication with a totally positive matrix increases the size of the numbers. Geometrically, the limiting position of the planes in the sequence defined above corresponds to moving from the plane spanned by the first $m$ standard basis vectors to the so-called opposite flag, spanned by the last $m$ standard basis vectors. For example, in Figure 2 , we see the first and last line in the sequence as those lines spanned by the corners of the tetrahedron. With a localization as in (4-1) the intersection condition for the opposite flag is

$$
\operatorname{det}\left[\begin{array}{cccc}
1 & 0 & x_{11} & x_{12} \\
0 & 1 & x_{21} & x_{22} \\
0 & 0 & 1 & 0 \\
0 & 0 & 0 & 1
\end{array}\right]=0
$$

which reduces to $1=0$. This geometric interpretation exhibits the extremal cases of numerical troubles with this localization. So we use a localization that has in every row and column at least one free variable to represent any point of intersection. The expansion leads to polynomials of degree $p$.

Whitney's reduction theorem [1952], in the form of Loewner [1955], is used to generate the upper triangular totally positive matrices. For every $n$-by- $n$ matrix we generate $\frac{1}{2} n(n-1)$ positive random numbers. Tuning the size of those random numbers allows us to control the speed by which the sequence 
moves to the opposite flag. There is the following tradeoff. A selection of small random numbers leads to input planes that lie too close to each other, whereas with large numbers we end up with a cluster of planes too close to the opposite flag. The actual input to the cheater's homotopy consists in the orthonormal part of the QR-factorization applied to the generated sequence of $m$-planes.

The case $(3,4)$ verified by the SAGBI homotopies has total degree $D=4^{12}=16,777,216$ and 4-homogeneous Bézout number $B=369,600$ with the partition $\left\{\left\{x_{11}, x_{51}, x_{71}\right\},\left\{x_{22}, x_{62}, x_{72}\right\},\left\{x_{33}, x_{63}, x_{73}\right\}\right.$, $\left.\left\{x_{44}, x_{64}, x_{74}\right\}\right\}$, whereas we know there are only 462 solutions. All 462 solutions are different from each other, with condition numbers less than $10^{8}$, and as conjectured, are all real. The components of the solution $p$-planes all have the same sign pattern. The matrices that represent those solutions are not totally positive, although all corresponding minors have the same sign.

\section{AVAILABLE SOFTWARE AND EXECUTION TIMES}

This section describes programming and computational experiences with the author's software PHC (Polynomial Homotopy Continuation). See [Verschelde 1999] for a complete description of PHC.

\section{A. A new driver added to PHC}

Homotopy continuation methods have two parts:

1. Bounds for the number of roots are obtained by applying the theorems of Bézout or Bernshteĭn. For sparse systems, the mixed volume yields a much sharper bound and polyhedral homotopies are invoked to solve a start system with random coefficients. For general dense systems or when the degrees are used to count the roots, the construction of a start system does not require continuation.

2. The path tracker exploits the fact that solution paths of polynomial homotopies do not turn back, so that the continuation parameter is fixed during the correction stage. Path crossing is avoided enforcing Newton's method to converge quadratically when correcting the solutions. At the end of the solutions paths, condition numbers are computed and mathematical certificates of divergence [Huber and Verschelde 1998] can be obtained.
The implemented homotopy methods are powerful and reliable enough to operate as a general-purpose solver in black-box mode for small and mediumsized problems. This is illustrated in [Verschelde 1999] on the test suite of about eighty polynomial systems from a wide variety of sources that are in the distribution of the package. Of course, given the poor performance of the standard root counts, the black-box solver is not recommended for the $(2,8)$ problem.

The SAGBI homotopies are implemented in a separate module of $\mathrm{PHC}$, which generates the equations, calls the dynamic lifting algorithm and the path-tracking routines. Besides the driver, the new implementation consists of Laplace expansion, the assignment of the powers of the continuation parameter in the flat deformation, and the generation of the osculating planes. The new public version of PHC offers this driver when calling phc -e. Another new feature in PHC is the multi-precision arithmetic to evaluate and refine the solutions of a polynomial system.

\section{B. Computational Experiences}

In Table 3 the timing results for the main computation are summarized. Calculations are done on a $166 \mathrm{MHz}$ Pentium II processor with $64 \mathrm{Mb}$ internal memory running Linux.

In Table 3 we see that the most expensive stage is the cheater's homotopy to a specific real problem instance. The $(4,3)$-case is given to illustrate how the computations scale up when going from a 12dimensional to a 16-dimensional system, from 462 to 1430 roots. Dividing total time by 1430 paths, the cost for each path is about 1 minute user CPU time. The $(3,4)$-case with totally positive matrices is harder because of the fourth-degree polynomials.

The systems with their solutions are available via the author's web page. The condition numbers of the Jacobian matrices evaluated at the end of the paths range between $10^{4}$ and $10^{8}$, which is in the worst-case scenario still sufficient to guarantee the correctness to eight decimal places. The distance between two roots is at least $10^{-8}$.

Moreover, these condition numbers are complex in the sense that they reflect the sensitivity of perturbations with complex numbers. The real condition numbers must be much smaller, because the largest 


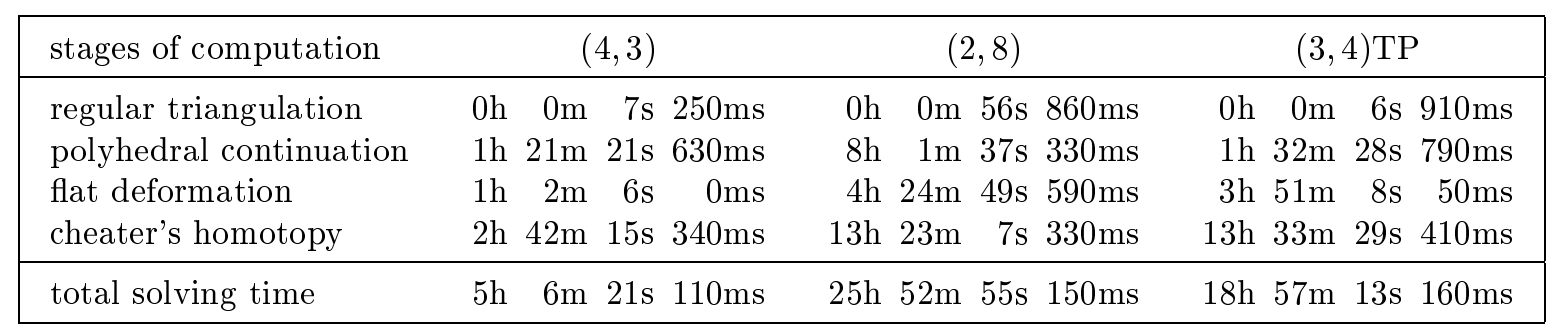

TABLE 3. User CPU times for solving the cases $(4,3),(2,8)$, and $(3,4)$ with total positivity.

imaginary parts of the solutions are of order $10^{-20}$. After application of Newton's method (using QR decomposition followed by least squares approximation to solve the linear systems) ten times to each solution, the imaginary parts shrunk below $10^{-100}$.

\section{CONCLUSIONS}

For the $(2,8)$-case we computed a polynomial system with all 1430 solutions real, well-conditioned and distinct from each other. Homotopy continuation extends this real problem instance to a manifold of polynomial systems that have all their solutions real.

The results of the calculations described in this paper are relevant for the following reasons:

1. The class of systems constitutes an interesting benchmark for polynomial system solvers. We can exploit the synthetic nature of enumerative geometry questions to choose the most favorable numerical representation of the problem.

2. In addition to providing computational evidences for conjecture in real algebraic geometry, these tools and experiences may become useful to the engineering community. Providing stable numerical algorithms for the pole placement problem is one of open problems listed in [Rosenthal and Willems 1999]. This paper shows that homotopy continuation is a first promising step.

3. Almost all computations in algebraic geometry are exact as implemented in computer algebra systems. The calculations reported in this paper show that meaningful results (in the spirit of [Stetter 1998]) are obtained from numerical approximations.

4. The practical efficiency of homotopies for solving polynomial systems that arise from expanding determinants is demonstrated. How to apply the theory of [Dedieu and Shub 2000] to the overdetermined homotopies that arise in the Pieri homotopies [Huber et al. 1998] is an interesting future research project.

Finally we remark that the Pieri homotopies presented in [Huber et al. 1998] have been implemented in [Huber and Verschelde 2000]. Those Pieri homotopies have been extended to compute the feedback laws of the dynamic pole pole placement problem, which is equivalent [Rosenthal 1994] to enumerating all curves of degree $q$ that produce $p$-planes which meet $m p+q(m+p)$ given $m$-planes in $\mathbb{C}^{m+p}$ at specified interpolation points. One analogue of the Shapiro conjectures for the case $q>0$ does not hold; see [Sottile 2000a] for a counterexample.

\section{ACKNOWLEDGEMENTS}

I thank Birk Huber for explaining flat deformations to me in his apartment in Princeton and for his help in studying the problem of the balancing pointer. Frank Sottile told me about the 'osculating normal curves', which triggered the idea of using Chebyshev polynomials; also his explanation of 'opposite flags' was most inspiring. Frank made several helpful comments on the first draft of this paper. I am grateful to him and to Bernd Sturmfels for encouraging the implementation and calculations. I thank Marie-Françoise Roy for pointing to the property of random real polynomials in the Chebyshev basis. The discussions with T. Y. Li and Xiaoshen Wang on cheater's homotopy were most fruitful. I thank Joachim Rosenthal for writing down for me the connections (5-5) between the equations in the pole placement problem. Last and certainly not least the special programs at MSRI in the fall of 1998 brought us together, which largely contributed to the writing of this paper. 


\section{REFERENCES}

[Allgower and Georg 1990] E. L. Allgower and K. Georg, Numerical continuation methods: an introduction, Springer Ser. Comput. Math. 13, Springer, Berlin, 1990.

[Allgower and Georg 1997] E. L. Allgower and K. Georg, "Numerical path following", pp. 3-207 in Handbook of numerical analysis, $V$ : Techniques of scientific computing (Part 2), edited by P. G. Ciarlet and J. L. Lions, North-Holland, Amsterdam, 1997.

[Ando 1987] T. Ando, "Totally positive matrices", Linear Algebra Appl. 90 (1987), 165-219.

[Bernshteĭn 1975] D. N. Bernshtel̆n, "The number of roots of a system of equations", Funktsional. Anal. $i$ Prilozhen. 9:3 (1975), 1-4. In Russian; translated in Functional Anal. Appl., 9:3 (1975), 183-185.

[Bharucha-Reid and Sambandham 1986] A. T. BharuchaReid and M. Sambandham, Random polynomials, Academic Press Inc., Orlando, FL, 1986.

[Blum et al. 1998] L. Blum, F. Cucker, M. Shub, and S. Smale, Complexity and real computation, Springer, New York, 1998.

[Brockett and Byrnes 1981] R. W. Brockett and C. I. Byrnes, "Multivariable Nyquist criteria, root loci, and pole placement: a geometric viewpoint", IEEE Trans. Automat. Control 26:1 (1981), 271-284.

[Byrnes 1989] C. I. Byrnes, "Pole assignment by output feedback", pp. 31-78 in Three decades of mathematical system theory, edited by $\mathrm{H}$. Nijmacher and J. M. Schumacher, Lecture Notes in Control and Inform. Sci. 135, Springer, Berlin, 1989.

[Cox et al. 1997] D. Cox, J. Little, and D. O'Shea, Ideals, varieties, and algorithms: An introduction to computational algebraic geometry and commutative algebra, 2nd ed., Undergraduate Texts in Math., Springer, New York, 1997.

[Cox et al. 1998] D. Cox, J. Little, and D. O'Shea, Using algebraic geometry, Graduate Texts in Math. 185, Springer, New York, 1998.

[Cucker and Roy 1990] F. Cucker and M.-F. Roy, "A theorem on random polynomials and some consequences in average complexity", J. Symbolic Comput. 10:5 (1990), 405-409.

[Dedieu and Shub 2000] J. P. Dedieu and M. Shub, "Newton's method for overdetermined systems of equations", Math. Comp. 69:231 (2000), 1099-1115.

[Edelman et al. 1999] A. Edelman, T. A. Arias, and S. T. Smith, "The geometry of algorithms with orthogonality constraints", SIAM J. Matrix Anal. Appl. 20:2 (1999), 303-353.

[Eisenbud 1995] D. Eisenbud, Commutative algebra with a view toward algebraic geometry, Graduate Texts in Math. 150, Springer, New York, 1995.

[Faugère et al. 1998] J.-C. Faugère, F. Rouillier, and P. Zimmerman, 1998. Personal communication.

[Fulton 1997] W. Fulton, Young tableaux, London Math. Soc. Student Texts 35, Cambridge University Press, Cambridge, 1997.

[Gerald 1978] C. F. Gerald, Applied numerical analysis, 2nd ed., Addison-Wesley, Reading, MA, 1978.

[Golub and Van Loan 1996] G. H. Golub and C. F. Van Loan, Matrix computations, 3rd ed., Johns Hopkins Univ. Press, Baltimore, 1996.

[Huber and Sturmfels 1995] B. Huber and B. Sturmfels, "A polyhedral method for solving sparse polynomial systems", Math. Comp. 64:212 (1995), 1541-1555.

[Huber and Verschelde 1998] B. Huber and J. Verschelde, "Polyhedral end games for polynomial continuation", Numer. Algorithms 18:1 (1998), 91-108.

[Huber and Verschelde 2000] B. Huber and J. Verschelde, "Pieri homotopies for problems in enumerative geometry applied to pole placement in linear systems control", SIAM J. Control Optim. 38:4 (2000), 12651287.

[Huber et al. 1998] B. Huber, F. Sottile, and B. Sturmfels, "Numerical Schubert calculus", J. Symbolic Comput. 26:6 (1998), 767-788.

[Kailath 1980] T. Kailath, Linear systems, Prentice-Hall Inc., Englewood Cliffs, NJ, 1980.

[Kushnirenko 1976] A. G. Kushnirenko, "Newton polytopes and the Bézout theorem", Funktsional. Anal. $i$ Prilozhen. 10:3 (1976), 82-83. In Russian; translated in Functional Anal. Appl. 10:3 (1976), 233-235.

[Li 1997] T. Y. Li, "Numerical solution of multivariate polynomial systems by homotopy continuation methods", pp. 399-436 in Acta numerica, 1997, Cambridge Univ. Press, Cambridge, 1997.

[Li and Wang 1992] T. Y. Li and X. S. Wang, "Nonlinear homotopies for solving deficient polynomial systems with parameters", SIAM J. Numer. Anal. 29:4 (1992), 1104-1118.

[Li et al. 1989] T. Y. Li, T. Sauer, and J. A. Yorke, "The cheater's homotopy: an efficient procedure for solving systems of polynomial equations", SIAM J. Numer. Anal. 26:5 (1989), 1241-1251. 
[Loewner 1955] C. Loewner, "On totally positive matrices", Math. Z. 63 (1955), 338-340.

[Morgan 1987] A. Morgan, Solving polynomial systems using continuation for engineering and scientific problems, Prentice-Hall Inc., Englewood Cliffs, NJ, 1987.

[Morgan and Sommese 1987] A. Morgan and A. Sommese, "A homotopy for solving general polynomial systems that respects $m$-homogeneous structures", Appl. Math. Comput. 24:2 (1987), 101-113.

[Morgan and Sommese 1989] A. P. Morgan and A. J. Sommese, "Coefficient-parameter polynomial continuation", Appl. Math. Comput. 29:2(II) (1989), 123-160. Errata in 51:2-3 (1992), 207.

[Rosenthal 1994] J. Rosenthal, "On dynamic feedback compensation and compactification of systems", SIAM J. Control Optim. 32:1 (1994), 279-296.

[Rosenthal and Schumacher 1997] J. Rosenthal and J. M. Schumacher, "Realization by inspection", IEEE Trans. Automat. Control 42:9 (1997), 1257-1263.

[Rosenthal and Sottile 1998] J. Rosenthal and F. Sottile, "Some remarks on real and complex output feedback", Systems Control Lett. 33:2 (1998), 73-80. See http://www.nd.edu/ rosen/pole for a description of computational aspects of the paper.

[Rosenthal and Willems 1999] J. Rosenthal and J. C. Willems, "Open problems in the area of pole placement", pp. 181-191 in Open problems in mathematical systems and control theory, edited by V. D. Blondel et al., Comm. and Control Engin. Series, Springer, London, 1999.

[Schubert 1891] H. Schubert, "Beziehungen zwischen den linearen Räumen auferlegbaren charakteristischen Bedingungen", Math. Ann. 38 (1891), 588-602.

[Sottile 1999] F. Sottile, "The special Schubert calculus is real", Electron. Res. Announc. Amer. Math. Soc. 5 (1999), 35-39. See http://www.ams.org/era.

[Sottile 2000a] F. Sottile, "Real rational curves in Grassmannians", J. Amer. Math. Soc. 13:2 (2000), 333-341.

[Sottile 2000b] F. Sottile, "Real Schubert calculus: polynomial systems and a conjecture of Shapiro and Shapiro", Experiment. Math. 9:2 (2000), 161-182.
[Stetter 1998] H. J. Stetter, "Numerical polynomial algebra", Technical report, 1998. Notes for the tutorial Interaction between Numerical Analysis and Computer Algebra, held at ISSAC'98, Rostock, Germany.

[Sturmfels 1993] B. Sturmfels, Algorithms in invariant theory, Springer, Vienna, 1993.

[Sturmfels 1996] B. Sturmfels, Gröbner bases and convex polytopes, Univ. Lecture Ser. 8, Amer. Math. Soc., Providence, RI, 1996.

[Sturmfels 1998] B. Sturmfels, "Polynomial equations and convex polytopes", Amer. Math. Monthly 105:10 (1998), 907-922.

[Verschelde 1999] J. Verschelde, "Algorithm 795: PHCpack: A general-purpose solver for polynomial systems by homotopy continuation", ACM Trans. Math. Software 25:2 (1999), 251-276. See http://www.math. msu.edu/ jan.

[Verschelde et al. 1994] J. Verschelde, P. Verlinden, and R. Cools, "Homotopies exploiting Newton polytopes for solving sparse polynomial systems", SIAM J. Numer. Anal. 31:3 (1994), 915-930.

[Verschelde et al. 1996] J. Verschelde, K. Gatermann, and R. Cools, "Mixed-volume computation by dynamic lifting applied to polynomial system solving", Discrete Comput. Geom. 16:1 (1996), 69-112.

[Wampler 1992] C. W. Wampler, "Bezout number calculations for multi-homogeneous polynomial systems", Appl. Math. Comput. 51:2-3 (1992), 143-157.

[Wampler et al. 1990] C. W. Wampler, A. P. Morgan, and A. J. Sommese, "Numerical continuation methods for solving polynomial systems arising in kinematics", ASME J. of Mechanical Design 112:1 (1990), 59-68.

[Wampler et al. 1992] C. W. Wampler, A. P. Morgan, and A. J. Sommese, "Complete solution of the nine-point path synthesis problem for four-bar linkages", ASME J. of Mechanical Design 114:1 (1992), 153-159.

[Whitney 1952] A. M. Whitney, "A reduction theorem for totally positive matrices", J. Analyse Math. 2 (1952), 88-92.

[Yakoubsohn 1999] J.-C. Yakoubsohn, "Finding zeros of analytic functions: $\alpha$-theory for secant type methods", J. Complexity 15:2 (1999), 239-281.

Jan Verschelde, Department of Mathematics, Statistics, and Computer Science, University of Illinois at Chicago, 851 S. Morgan (M/C 249), Chicago, IL 60607 (jan.verschelde@na-net.ornl.gov)

Received December 1, 1998; accepted May 4, 1999 